\title{
Local-scale determinants of arboreal spider beta diversity in a temperate forest: roles of tree architecture, spatial distance, and dispersal capacity
}

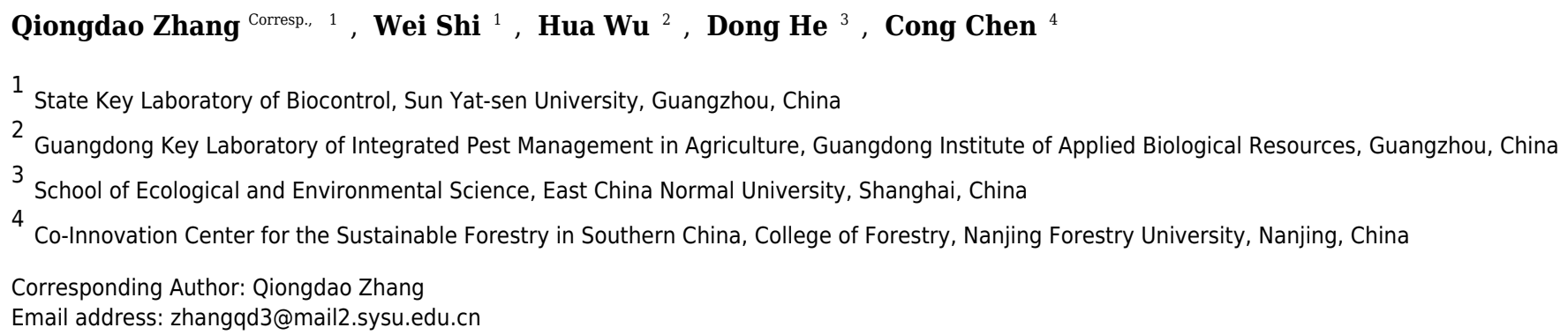

Spiders are a functionally important taxon in forest ecosystems, but the determinants of arboreal spider beta diversity are poorly understood at the local scale. We examined spider assemblages in 324 European beech (Fagus sylvatica) trees of varying sizes across three forest stands in Würzburg (Germany) to disentangle the roles of tree architecture, spatial distance, and dispersal capacity on spider turnover across individual trees. A large proportion of tree pairs (66\%) showed higher compositional dissimilarity in spider assemblages than expected by chance, suggesting prominent roles of habitat specialization and/or dispersal limitation. Trees with higher dissimilarity in DBH and canopy volume, and to a lesser extent in foliage cover, supported more dissimilar spider assemblages, suggesting that tree architecture comprised a relevant environmental gradient of sorting spider species. Variation partitioning revealed that $28.4 \%$ of the variation in beta diversity was jointly explained by tree architecture, spatial distance (measured by principal coordinates of neighbor matrices) and dispersal capacity (quantified by ballooning propensity). Among these, dispersal capacity accounted for a comparable proportion as spatial distance did (6.8\% vs. 5.9\%). Beta diversity did not significantly differ between high- and low-vagility groups, but beta diversity in species with high vagility was more strongly determined by spatially structured environmental variation. Altogether, both niche specialization, along the environmental gradient defined by tree architecture, and dispersal limitation are responsible for structuring arboreal spider assemblages. High dispersal capacity of spiders appears to reinforce the role of nicherelated processes. 
1 Local-scale determinants of arboreal spider beta diversity in a

2 temperate forest: roles of tree architecture, spatial distance, and

3 dispersal capacity

4

Qiongdao Zhang ${ }^{*}$, Wei Shi ${ }^{1}$, Hua $\mathrm{Wu}^{2}$, Dong He ${ }^{3}$, Cong Chen ${ }^{4}$

6

$7 \quad{ }^{1}$ SYSU-Alberta Joint Lab for Biodiversity Conservation, State Key Laboratory of Biocontrol, Sun Yat-sen

University, Guangzhou 510275, China

${ }^{2}$ Guangdong Key Laboratory of Integrated Pest Management in Agriculture, Guangdong Institute of Applied

Biological Resources, Guangzhou 510260, China

${ }^{3}$ School of Ecological and Environmental Science, East China Normal University, Shanghai 200241, China

${ }^{4}$ Co-Innovation Center for the Sustainable Forestry in Southern China, College of Forestry, Nanjing Forestry

University, Nanjing, 210037, China

*Correspondence: Qiongdao Zhang,SYSU-Alberta Joint Lab for Biodiversity Conservation, State Key Laboratory

of Biocontrol, Sun Yat-sen University, Guangzhou 510275, PR China

Email: zhangqd3@mail2.sysu.edu.cn (Q.Zhang)

Tel/Fax number: +861562626 6938; +86 02084111544 


\section{ABSTRACT}

20 Spiders are a functionally important taxon in forest ecosystems, but the determinants of arboreal spider

21 beta diversity are poorly understood at the local scale. We examined spider assemblages in 324 European beech (Fagus sylvatica) trees of varying sizes across three forest stands in Würzburg (Germany) to disentangle the roles of tree architecture, spatial distance, and dispersal capacity on spider turnover across individual trees. A large proportion of tree pairs (66\%) showed higher compositional dissimilarity in spider assemblages than expected by chance, suggesting prominent roles of habitat specialization and/or dispersal limitation. Trees with higher dissimilarity in DBH and canopy volume, and to a lesser extent in foliage cover, supported more dissimilar spider assemblages, suggesting that tree architecture comprised a relevant environmental gradient of sorting spider species. Variation partitioning revealed that $28.4 \%$ of the variation in beta diversity was jointly explained by tree architecture, spatial distance (measured by principal coordinates of neighbor matrices) and dispersal capacity (quantified by ballooning propensity). Among these, dispersal capacity accounted for a comparable proportion as spatial distance did (6.8\% vs. 5.9\%). Beta diversity did not significantly differ between high- and low-vagility groups, but beta diversity in species with high vagility was more strongly determined by spatially structured environmental variation. Altogether, both niche specialization, along the environmental gradient defined by tree architecture, and dispersal limitation are responsible for structuring arboreal spider assemblages. High dispersal capacity of spiders appears to reinforce the role of niche-related processes. 
INTRODUCTION

Beta diversity, defined as the compositional dissimilarity between species assemblages, is an important component of biodiversity (Whittaker 1960; Anderson et al. 2011). Beta diversity considers valuable information about how biodiversity is spatially organized and provides a link between local and regional patterns, thereby serving as a key conceptual tool for understanding species distributions and community assembly (Anderson et al. 2011). Furthermore, exploring the patterns and causes of beta diversity could provide valuable insights for biodiversity conservation and management (Socolar et al. 2016). To date, beta diversity studies have been largely biased towards plants and birds (Socolar et al. 2016), whereas spiders, another functionally important taxon, have been relatively less studied in this respect (Carvalho et al. 2011; Rodriguez-Artigas et al. 2016).

Spiders are a functionally important taxon in terrestrial ecosystems (Riechert 1974; Wise 1993). They consume a large variety of prey and respond readily to vegetation changes (Riechert 1974; Riechert \& Lockley 1984; Gómez et al. 2016). Therefore, spiders may serve as a useful indicator of environmental quality and overall biodiversity in an ecosystem (Willett 2001). In forests, spiders can achieve high levels of local species richness and abundance from the litter layers to the canopy (Basset 2001). As such, it is important to identify the determinants of arboreal spider diversity to better understand the mechanism of spider community assembly and inform biological conservation in forests. Previous studies have suggested that environmental heterogeneity, spatial distance and/or dispersal capacity differences can be relevant for beta diversity in general (Jiménez-Valverde et al. 2010; Carvalho et al. 2011; RodriguezArtigas et al. 2016). However, the influence of these factors on beta diversity of arboreal spiders is still unclear. 
According to niche theory, which assumes habitat specialization as an important driver of species turnover, environmental heterogeneity is the most relevant predictor of beta diversity (Gilbert \& Lechowicz 2004; Legendre et al. 2009a). For spiders, structural complexity of vegetation, which affects the availability of web attachment structures (Rypstra et al. 1999) and favored prey (Harwood et al. 2003), has been recognized as an important environmental factor influencing their community composition and diversity at the stand level (Hajalet al. 1998, 2000; Jiménez-Valverde \& Lobo 2007; Carvalho et al. 2011; Rodriguez-Artigas et al. 2016). However, the influence of individual plant architecture on arboreal spider beta diversity is poorly understood. An investigating of this influence may help elucidate lower-level processes regulating diversity. Since trees, especially their canopies, provide arboreal spiders with sites for web attachment, foraging, oviposition, and shelter (Hajalet al. 2000; Hsieh 2011), the architecture of individual tree may serve as the immediate habitat template for spider assemblages in tree canopies (Halaj et al. 2000). We hence predict that tree pairs with a more similar architecture will support a more similar species composition.

By definition, beta diversity integrates the spatial component dimension, thus spatial distance is also proposed as an important predictor of beta diversity (Soininen et al. 2007). With the rise of neutral theory, which highlights the role of dispersal limitation (Hubbell 2001), the importance of spatial distance in predicting species distribution is increasingly recognized. Because spatial and environmental factors usually interact with each other, much effort (Tuomisto et al. 2003; Anderson et al. 2011) has been invested towards partitioning the variation in species beta diversity into pure spatial component, pure environmental component and spatially structured environmental component (i.e., the interaction between space and environments, Fig. 1a). The distinction between spatial and environmental influences on 
diversity is of paramount importance to community assembly studies because it provides insight into the underlying processes that create patterns of beta diversity (Legendre et al. 2009a; Jiménez-Valverde et al. 2010; Carvalho et al. 2011).

The relative contribution of spatial distance to beta diversity are often assumed indicating the importance of dispersal limitation (Jiménez-Valverde et al. 2010), but dispersal capacity is actually not equal across species. Dispersal limitation is more likely a result from the interaction between spatial distance and dispersal capacity. Therefore, it would be helpful to disentangle the relative roles of spatial distance and dispersal capacity in shaping beta diversity. While dispersal capacity is difficult to quantify in many taxa (Thomas et al. 2003; Werth et al. 2006; Riginos et al. 2009), it has been shown to well relate to ballooning propensity in spiders (Thomas et al. 2003; Jiménez-Valverde et al. 2010). Variation in body size and ecological strategy among spider species results in a high diversity of ballooning propensity, a characteristic passive air dispersal using silk threads (Dean \& Sterling 1985; Richardson et al. 2006). We hence expect that ballooning propensity has a comparative contribution to spider beta diversity as spatial distance.

Furthermore, the distribution of a highly mobile species is likely more dependent on environmental than on spatial factors, as these spiders are able to move from unfavorable to favorable habitats. A less mobile species may be more restrained by spatial distance because of their inability to disperse to distant optimal environments (Araújo \& Pearson 2005). If a more mobile species coincides with a wideoccupancy species, we predict that spatially structured environmental variables will be a major determinant of spider beta diversity for the more mobile species. 
The present study focuses on spider beta diversity pattern in a temperate deciduous forest in Germany,

which is dominated by European beech (Fagus sylvatica). Specifically, we attempted to answer to the

following questions: (i) How does individual tree architecture modify arboreal spider beta diversity? (ii)

What is the relative importance of tree architecture, spatial distance and dispersal capacity on beta

diversity? (iii) Do spiders with varying dispersal capacity show different beta diversity patterns?

\section{MATERIALS AND METHODS}

\section{Study area}

We used a dataset collected by Hsieh (2011) at the Würzburg University Forest (5001' N, $\left.10^{\circ} 30^{\prime} \mathrm{E}\right)$ in

Germany. The forest covers about 2664 ha, $18 \%$ of which is classified as "High Conservation Value

Forest" by the Forest Stewardship Council. Mean yearly temperature and precipitation are $7.5^{\circ} \mathrm{C}$ and 675

$\mathrm{mm}$, respectively, in this region. European beech (F. sylvatica, nearly $30 \%$ of abundance) is the most

common tree species inthe study area, followed by oak (Quercusrobur, 19\%) and spruce (Piceaabies,

$12 \%)$. We focused on beech trees to investigate the composition, diversity and species turnover of

arboreal spiders.

114 Three forest stands with varying age and height characteristics were surveyed: old-growth beech

115 (>150 years old, 20-26 m tall), mature beech (50-60 years old, 13-19 m tall), and young beech (20-25

years old, 5-6 m tall). These three forest stands were distributed in proximity within a 6-ha area. Within

\section{Spider sampling}


120

121

122

123

124

125

126

127

128

129

130

131

132

133

134

135

136

137

138

139

140

From 2005 to 2007, beech trees were fogged for spider collection using a Swingfog (SN-50) machine, which emits a natural pyrethrum insecticide that is not toxic to terrestrial vertebrates. Each sampled tree was fogged three times during a given fogging event. As spiders fell from the trees, they were collected on plastic sheets covering $90 \%$ of the fogged tree's crown vertical projection area on the ground $\left(46 \mathrm{~m}^{2}\right.$ sheets for old-growth and mature beeches and $6 \mathrm{~m}^{2}$ sheets for young beeches). Fogging began at daybreak and lasted approximately 10 minutes at every fogging time with an interval of 50 minutes. Spider samples were collected from the plastic sheets two hours after the completion of the three foggings. A total of 33 beech trees produced no spider samples after fogging. Subsequently, we had assemblages of 86, 101, and 102 trees in the old-growth, mature and young stands, respectively. The spider samples were stored in bags with $70 \%$ alcohol for transport to the laboratory, where specimens were identified to the species level using species-specific attributes of the palpal organ or epigynum according to Heimer \& Nentwig (1991) and Roberts (1996). Specimens were named according to the nomenclature suggested by World Spider Catalog (2017).

To evaluate inventory completeness, the non-parametric estimator of species richness, Jack1, which represents species richness expected to be present in communities with highly heterogeneous sample coverage, was computedin R version 3.2.3 (R Development Core Team 2017) using the 'vegan' package

(Oksanen et al. 2015).

\section{Environmental and spatial variables}

Five architectural traits were measured for each individual tree, including diameter at breast height (DBH), total height $\left(\mathrm{H}_{\mathrm{t}}\right)$, clear-pole height $\left(\mathrm{H}_{\mathrm{cp}}\right)$, crown radius $(\mathrm{R})$, foliage cover $(\mathrm{FC})$ and canopy volume $(\mathrm{CV})$, as indicators of habitat structure available to spiders. Clear-pole heightis the distance from underground to 
the lowest branch of an individual tree. Canopy volume is estimated by crown radius multiplied by

canopy depth $\left(\mathrm{H}_{\mathrm{t}}-\mathrm{H}_{\mathrm{cp}}\right)$. These five architectural traits varied greatly between trees and comprised a clear environmental gradient along which spiders were likely sorted into disparate assemblages.

Spatial variables were computed using Principal Coordinates of Neighbor Matrices (PCNM), a spatial analysis method for the detection of spatial trends across a range of scales (Borcard et al. 2004). First, the spatial coordinates of each sampling tree wereused to build a Euclidean distance matrix. Then, this matrix was truncated to the smallest distance that keeps all trees connected in a single network, which corresponds to the maximum distance between two successive sampling trees in one-dimensional studies. Finally, a Principal Coordinate Analysis (PCoA) was conducted on the Euclidean distance matrix to generate 23 PCNM eigenvectors with positive eigenvalues. These PCNM variables were then used as explanatory variables to analyze the spatial variation of the spider community composition.

\section{Classification of spiders with different dispersal capacity}

Dispersal capacity varies widely among spider species and is tightly connected with ballooning propensity (Jiménez-Valverde et al. 2010). Previous studies have established ballooning propensity as the main method for evaluating dispersal capacity in spiders (Thomas et al. 2003; Jiménez-Valverde et al. 2010). We assembled ballooning propensity information for all species encountered in this study from the existing literature (Bell et al. 2005; Schirmel et al. 2012). Then we arranged the species into two categories according to their ballooning propensity: high- (where both adults and juveniles frequently balloon) and low- (where adults occasionally balloon but juveniles rarely balloon) vagility.

\section{Data analysis}


161 We utilized the Bray-Curtis dissimilarity index (Bray \& Curtis 1957) to calculate spider compositional

162 dissimilarities between trees using abundance data. We subsequently addressed whether the observed

163 species turnover differed from the random expectation by comparing the observed values with 1,000

164 values generated by a null model. The null model randomized the community matrix while maintaining

165 the observed species incidence across trees and the species richness in each tree (Gotelli 2000). In doing

166 so, we removed neutral sampling effects so as to test for the effects of underlying structured forces (e.g.,

habitat specialization and dispersal limitation). The standard effect size (SES) of non-random underlying

forces was calculated as the difference between the observed and randomly expected dissimilarity. A SES

value greaterthan zero indicates an identified effect of habitat specialization and/or dispersal limitation,

and the converse indicates the predominance of biotic homogenization (e.g., the loss of rare species,

widespread species invasion and/or the overwhelming occupancy of common species).

To avoid collinearity among tree architecture variables, we applied variation inflation factor (VIF)

values to eliminate redundant environmental variables (i.e., $\mathrm{H}_{t}$ and $\mathrm{H}_{\mathrm{cp}}$ ). After checking the length of the

dominant gradient in species composition $(\mathrm{L}=2.7)$, a redundancy analysis ( $\mathrm{RDA})$ was employed to

examine the relationship between spider composition and non-redundant tree architecture variables.

Forward selection (Blanchet et al. 2008) was used to identify explanatory variables that significantly

explain the variation in species composition $(\mathrm{P}<0.05$ after 1000 random permutations). This procedure

was also carried out for spatial (PCNMs) variables. Thirteen PCNM eigenvectors (i.e., PCNMs1-9, 12, 25,

29 and 23) were preserved by a forward selection procedure in RDA. 
distance, and community-level dispersal capacity dissimilarity. Community-level dispersal capacity dissimilarity was defined as the differences in vagility group composition between two communities in this study. Variation partitioning was carried out through a series of partial RDAs. Because the unadjusted $\mathrm{R}^{2}$ values were biased (Peres-Neto et al. 2006), the $\mathrm{R}^{2}$ values were adjusted to account for the explanatory variables. Negative adjusted $\mathrm{R}^{2}$ can be ignored (considered as null) for the ecological interpretation of the results. We then performed 9999 permutations testing for significance of unique fractions (i.e., tree architecture, spatial distance, dispersal capacity or their interactions) to determine how overall variation in species composition is partitioned among contrasting sets of explanatory variables. To evaluate the potential bias caused by the unmeasured variables in each stand, we also implemented variation partitioning in individual stands.

The beta diversity for each of the two dispersal capacity categories described above was calculated using the pair-wise dissimilarity measure based on the Bray-Curtis dissimilarity index. The dispersion of mean pair-wise dissimilarity values was estimated for each class by bootstrapping to detect significant differences between classes. One thousand bootstrap iterations were run, and the probability of obtaining lower mean pair-wise dissimilarity values for the high-vagility class in pair-wise comparisons by chance was calculated empirically. Likewise, variation partitioning analyses of beta diversity along environmental and spatial gradients were conducted for each spider dispersal capacity class.

The pair-wise dissimilarity measure was calculated using the 'betapart' package in $\mathrm{R}$ (Baselga et al. 2013a), whereas bootstrap iterations and comparisons were conducted using the 'boot' package (Canty \& Ripley 2015).The null model was run using the 'picante'package (Kembel et al. 2010); and PCNM eigenvectors were created using the 'PCNM'package (Legendre et al. 2009b). The forward selection, 
203

204

205

206

207

208

209

210

211

212

213

214

215

216

217

218

219

220

221

222

223

variation partitioning, and tests of significance of the fractions were computed in $\mathrm{R}$ version 3.2.3 using

the 'vegan' package (Oksanen et al. 2015).

\section{RESULTS}

A total of 7880 adult spider individuals belonging to 88 species, 61 genera and 16 families were collected throughout the survey from 2005 to 2007 . The species inventory completeness values for each stand and all stands pooled were generally high $(>73 \%$, Table 1). Therefore, we considered the inventories to be comparable among stands.

Across all possible tree pairs, about $66.4 \%$ of the Bray-Curtis spider compositional dissimilarity values were higher than expected by chance (Fig. 2). Only a small proportion of tree pairs had lowerthan-expected dissimilarity. Three tree architectural variables (i.e., DBH, CV and FC) were identified as significant predictors of spider composition, in which $14 \%$ of total variation was accounted for (Fig. 3).

Tree size (i.e., DBH and CV) defined the major axis of distinguishing the composition of spider assemblages, and FC characterized the second axis.

Variance partitioning revealed that $28.4 \%$ of the variation inspider assemblage dissimilarity was explained by tree architecture, spatial distance and dispersal capacity. A relatively large part of this variation was attributed to spatially structured environmental components $(8.7 \%)$, while $3.7 \%$, 5.9\%, and $6.8 \%$ were attributed to pure environmental components, pure spatial components, and pure dispersal capacity, correspondingly (Fig. $1 \mathrm{~b}$ and Table S1).

In each stand, the three factors jointly explained a similar proportion $(27 \%)$ of variation in spider composition (figure S1). However, two differences were detected in comparison with pooled analysis: (i) the importance of spatially structured environmental components dramatically diminished because of 
224 lower environmental variation within a stand than across stands; and (ii) the importance of dispersal

225 capacity varied greatly probably due to the compositional differences across three stands.

226 The mean pair-wise dissimilarity of the high-vagility spider class was slightly lower than the low-

227

228

vagility spider class but without statistical significance $(F=26.59, \mathrm{df}=999, P=1)$. Pure environmental

and spatial variables accounted for a similar proportion of the variation in compositional dissimilarity for both high- and low-vagility spider groups. Spatially-structured environmental factors explained twice as much variation in compositional dissimilarity for the high-vagility spider group as that for in the lowvagility spider group (Fig. 4 and Table S2).

\section{DISCUSSION}

Although it is difficult to sample the complete spider assemblage due to a large number of

rare species collected, according to the Jack 1 estimators, nearly $90 \%$ of the spider species expected to be

present in the beech forest canopies were captured in this study and more than $73 \%$ of these species were observed in each stand (Table 1). These results suggest inventories were adequate in this study, thereby enabling us to accurately quantify spider assemblage diversity within the beech forest. Nevertheless, it is important to keep awareness of some shortcomings associated with fogging. For example, some dead spiders might be suspended by their silk 'safety lines' and thus not be collected (Yanoviak et al. 2003); individuals with small body size might be trapped in minute droplets of spray in trees, or be blown away before being collected (Noyes and Sadka, 2003). In addition, due to daily air exchange patterns, fogging was mostly implemented at dawn and unable to capture nocturnal species (Hsieh 2011). Therefore, data collection via fogging could partly limit the generality of this study.

\section{Tree architecture as an environmental gradient}


245 We found that trees with dissimilar DBH and CV, and to a lesser extent FC, supported more dissimilar

246 spider assemblages. These factors explained about $14 \%$ of the variation in spider composition. Similarly,

247 Halaj et al. $(1998,2000)$ showed that leaf density and branching structure regulated the abundance and

248 distribution of arboreal spiders. Altogether, tree architecture comprises a relevantly environmental

249 gradient along which arboreal spiders are sorted into disparate assemblages in terms of their niche

250 specialization.

251 Several studies have identified vegetation structure as a key habitat signals of spider communities

252 (Carvalho et al. 2011; Gómez et al. 2016; Rodriguez-Artigas et al. 2016). Here we showed that lower-

level variables of vegetation structure (i.e., individual-tree architectural traits) also influence arboreal

spider composition. These results collectively endorse that non-trophic habitat heterogeneity within and

among forest stands acts as a "bottom-up template" for structuring spider assemblages (Halaj et al. 2000).

256 The importance of environmental constraints and dispersal limitation

The null model analysis showed that observed dissimilarity for most spider assemblage pairs was higher

than expected by chance, indicating a higher overall species turnover rate than randomly expected across individual trees. This pattern in beta diversity probably reflects prominent effects of habitat specialization and/or dispersal limitation (Tuomisto et al. 2003; Anderson et al. 2011). In contrast, biotic

homogenization appears to play a subordinate role, if any, in structuring spider assemblages in this study.

Our results showed that a combination of tree architecture, spatial distance and dispersal capacity explained a considerable proportion of the variation in beta diversity, and these three components represented a comparable proportion to each other. These results support the widely-held opinion that 
265

266

267

268

269

270

271

272

273

274

275

276

277

278

279

280

281

282

283

284

285

niche-based and dispersal processes are not mutually exclusive, but may work together to influence

species diversity and coexistence (Gilbert \& Lechowicz 2004; Legendre et al. 2009a).

\section{Influences of dispersal capacity on local beta diversity}

Our results indicate that dispersal capacity also plays a role in shaping beta diversity as spatial distance, suggesting that both of these factors should be considered when evaluating the importance of dispersal limitation. Interestingly, we found the mean pairwise compositional dissimilarity between sampling units (i.e., individual trees) was similar between two dispersal capacity classes within a confined spatial range.

Furthermore, we found little support for heavy spatial effects on beta diversity patterns of low-vagility spiders, suggesting that spatial autocorrelation in spider composition is not necessarily higher for lowvagility spiders. These findings suggest that the negative relationship between species turnover level and dispersal capacity found at large geographic scales (Jiménez-Valverde et al. 2010; Rodriguez-Artigas et al. 2016) may not be readily generalized at local scales.

Notably, we discovered that spatially structured environmental factors represented a far more important determinant of beta diversity for the high-vagility spider group than that for the low-vagility group. Due to the more pronounced spatial structure of environmental variation at the broad scale (pooled analysis in Figure 1) than those at the fine scale (individual stands in Figure S1), it seems that the distribution of high-vagility spiders is more responsive to the broad-scale environmental variation. In other words, highvagility helps promote habitat tracking (Araújo \& Pearson 2005). In contrast, low-vagility spiders appear

to live in a narrow niche, and be unable to adapt to broad-scale environmental variation $(\mathrm{r}=0.126 \pm 0.124$, polychoric correlation test between species frequency and vagility classes). As a result, dispersal capacity interacts with niche breadth and spatial scale to regulate spider beta diversity. 
286 CONCLUSIONS

287 Our results suggest both niche specialization, along with the environmental gradient defined by tree architecture, and dispersal limitation are responsible for structuring arboreal spider assemblages. In light of the importance of tree architecture, especially tree size that is a relevant environmental gradient for sorting arboreal spider species, stand structural heterogeneity is of great value in conserving spider biodiversity. For example, multi-cohort forests can provide more varied niches to promote species coexistence. In addition, less vagile spiders may be seen as rare species and be more affected by stochasticity. In this way, their higher conservation value is justified, but followed by a challenge of conserving these species with low predictability. which population dynamics are primarily driven by ecological drift and habitat independence (Legendre et al. 2009a). Additionally, some important variables, such as food sources and micro-climate factors that may be responsible for the assembly of spider communities in these trees, were not measured in this study, which may also contribute to the large variation.

\section{ACKNOWLEDGEMENTS}

We thank Yu-Lung Hsiehand Karl Eduard Linsenmair, who provided the dataset for this study. We are grateful to Fangliang He and Chengjin Chu of the SYSU-Alberta Joint Lab for Biodiversity Conservation, 
306 his assistance in data analysis. We thank Christine Verhilleat the University of British Columbia for

307 technical editing on an early version of the manuscript. 
309

310

311

312

313

314

315

316

317

318

319

320

321

322

323

324

325

326

327

328

329

330

331

332

333

334

335

336

337

338

339

340

341

342

343

344

345

346

347

\section{REFERENCES}

Anderson MJ, Crist TO, Chase JM, Vellend M, Inouye BD, Freestone AL, Sanders NJ, Cornell HV, Comita LS, and Davies KF. 2011. Navigating the multiple meanings of $\beta$ diversity: a roadmap for the practicing ecologist. Ecology letters 14:19-28.

Araújo MB, and Pearson RG. 2005. Equilibrium of species' distributions with climate. Ecography 28:693-695.

Baselga A, Orme D, Villeger S, Bortoli JD, and Leprieur F. 2013a. betapart: Partitioning beta diversity into turnover and nestedness components.

Basset Y. 2001. Invertebrates in the canopy of tropical rain forests How much do we really know? Tropical forest canopies: ecology and management: Springer, 87-107.

Bell J, Bohan D, Shaw E, and Weyman G. 2005. Ballooning dispersal using silk: world fauna, phylogenies, genetics and models. Bulletin of entomological research 95:69-114.

Blanchet FG, Legendre P, and Borcard D. 2008. Forward selection of explanatory variables. Ecology 89:2623-2632.

Borcard D, Legendre P, Avois-Jacquet C, and Tuomisto H. 2004. Dissecting the spatial structure of ecological data at multiple scales. Ecology 85:1826-1832.

Borcard D, Legendre P, and Drapeau P. 1992. Partialling out the spatial component of ecological variation. Ecology 73:1045-1055.

Bray JR, and Curtis JT. 1957. An ordination of the upland forest communities of southern Wisconsin. Ecological Monographs 27:325-349.

Canty A, and Ripley B. 2015. boot: Bootstrap R (S-Plus) Functions.

Carvalho JC, Cardoso P, Crespo LC, Henriques S, Carvalho R, and Gomes P. 2011. Determinants of beta diversity of spiders in coastal dunes along a gradient of mediterraneity. Diversity and Distributions 17:225-234.

Dean D, and Sterling W. 1985. Size and phenology of ballooning spiders at two locations in eastern Texas. Journal of Arachnology:111-120.

Gotelli N J. 2000.Null model analysis of species co - occurrence patterns. Ecology 81: 2606-2621.

Gómez JE, Lohmiller J, and Joern A. 2016. Importance of vegetation structure to the assembly of an aerial webbuilding spider community in North American open grassland. Journal of Arachnology 44:28-35.

Gilbert B, and Lechowicz MJ. 2004. Neutrality, niches, and dispersal in a temperate forest understory. Proc Natl Acad Sci U S A 101:7651-7656.

Halaj J, Ross DW, Moldenke AR. 1998. Habitat structure and prey availability as predictors of the abundance and community organization of spiders in western Oregon forest canopies. Journal of Arachnology26: 203-220.

Halaj J, Ross DW, and Moldenke AR. 2000. Importance of habitat structure to the arthropod food - web in Douglas - fir canopies. Oikos 90:139-152.

Harwood JD, Sunderland KD, and Symondson WO. 2003. Web - location by linyphiid spiders: prey - specific aggregation and foraging strategies. Journal of Animal Ecology 72:745-756.

Heimer S, and Nentwig W. 1991. Spinnen Mitteleuropas: Ein Bestimmungsbuch. Berlin: Verlag Paul Parey.

Hsieh SYL. 2011.The diversity and ecology of the spider communities of European beech canopy. Dissertation. Würzburg: University of Würzburg.

Hubbell SP. 2001. The Unified Neutral Theory of Biodiversity and Biogeography. Princeton, New Jersey, USA: Princeton University Press. 
Jiménez-Valverde A, Baselga A, Melic A, and Txasko N. 2010. Climate and regional beta - diversity gradients in spiders: dispersal capacity has nothing to say? Insect Conservation and Diversity 3:51-60.

Jiménez-Valverde A, and Lobo JM. 2007. Determinants of local spider (Araneidae and Thomisidae) species richness on a regional scale: climate and altitude vs. habitat structure. Ecological Entomology 32:113-122.

Kembel SW, Cowan PD, Helmus MR, Cornwell WK, Morlon H, Ackerly DD, Blomberg SP, and Webb CO. 2010. Picante: R tools for integrating phylogenies and ecology. Bioinformatics 26:1463-1464.

Legendre P, Borcard D, Blanchet FG, and Dray S. 2009b. PCNM: PCNM spatial eigenfunction and principle coordinate analyses.

Legendre P, Mi X, Ren H, Ma K, Yu M, Sun I, and He F. 2009a. Partitioning beta diversity in a subtropical broad - leaved forest of China. Ecology 90:663-674.

Oksanen J, Blanchet FG, Kindt R, Legendre P, Minchin PR, O'Hara RB, Simpson GL, Solymos P, Stevens MHH, and Wagner H. 2015. vegan: Community Ecology Package.

Peres-Neto PR, Legendre P, Dray S, and Borcard D. 2006. Variation partitioning of species data matrices: estimation and comparison of fractions. Ecology 87:2614-2625.

R Development Core Team. 2017. R: A language and environment for statistical computing. R Foundation for Statistical Computing, Vienna, Austria. URL http://www.R-project.org/.

Richardson B, Zabka M, Gray M, and Milledge G. 2006. Distributional patterns of jumping spiders (Araneae: Salticidae) in Australia. Journal of Biogeography 33:707-719.

Riechert SE. 1974. Thoughts on the Ecological Significance of Spiders. BioScience 24:352-356. 10.2307/1296741

Riechert SE, and Lockley T. 1984. Spiders as Biological Control Agents. Annual Review of Entomology 29:299-320. 10.1146/annurev.en.29.010184.001503

Roberts M. 1996. Spiders of Britain and Northern Europe. London, U. K: Collins.

Rodriguez-Artigas SM, Ballester R, and Corronca JA. 2016. Factors that influence the beta-diversity of spider communities in northwestern Argentinean Grasslands. PeerJ 4:e1946.

Rypstra AL, Carter PE, Balfour RA, and Marshall SD. 1999. Architectural features of agricultural habitats and their impact on the spider inhabitants. Journal of Arachnology:371-377.

Schirmel J, Blindow I, and Buchholz S. 2012. Life-history trait and functional diversity patterns of ground beetles and spiders along a coastal heathland successional gradient. Basic and Applied Ecology 13:606-614.

Socolar JB, Gilroy JJ, Kunin WE, and Edwards DP. 2016. How Should Beta-Diversity Inform Biodiversity Conservation? Trends in ecology \& evolution 31:67-80. https://doi.org/10.1016/j.tree.2015.11.005

Soininen J, McDonald R, and Hillebrand H. 2007. The distance decay of similarity in ecological communities. Ecography 30:3-12.

Thomas C, Brain P, and Jepson P. 2003. Aerial activity of linyphiid spiders: modelling dispersal distances from meteorology and behaviour. Journal of Applied Ecology 40:912-927.

Tuomisto H, Ruokolainen K, and Yli-Halla M. 2003. Dispersal, environment, and floristic variation of western Amazonian forests. Science 299:241-244.

Whittaker RH. 1960. Vegetation of the Siskiyou Mountains, Oregon and California. Ecological Monographs 30:279-338. 10.2307/1943563

Willett TR. 2001. Spiders and Other Arthropods as Indicators in Old-Growth Versus Logged Redwood Stands. Restoration Ecology 9:410-420. 10.1046/j.1526-100X.2001.94010.x

Wise DH. 1993. Spiders in ecological webs. Cambridge: Cambridge University Press. 
389

390

391

392

393

394

395

396

397

398

399

400

401

402

403

404

405

406

407

408

409

410

411

412

413

414

415

416

417

418

419

420

421

422

423

424

425

426

427

428

429
World Spider Catalog. 2017. Natural History Museum Bern, online at http://wsc.nmbe.ch, version 18.0, accessed on \{date of access \}. doi: 10.24436/2.

Yanoviak SP, Nadkarni NM, and Gering JC. 2003. Arthropods in epiphytes: a diversity component that is not effectively sampled by canopy fogging. Biodiversity \& Conservation 12:731-741. 
431 Table 1 Observed species richness, estimated species richness using non-parametric estimators (Jack1) and

432 inventory completeness of beech (F. sylvatica) stands.

Figure 1 Venn diagram showing relative influence of tree architecture, spatial distance and dispersal capacity on

beta diversity: (a) a conceptual representation of the contrast among environment, space, dispersal capacity and spatially-structured environmental components; (b) empirical variance partitioning of tree architecture, spatial distance, dispersal capacity effects on spider beta diversity.

Figure 2 The standard effect size of non-random processes on spider compositional dissimilarity (i.e., Bray-Curtis index) between trees. SES values above zero indicate that observed compositional dissimilarity is higher than expected by random assembly. The proportion of tree pairs with SES values above zero are $66.4 \%$.

441 Figure 3 The relationship between spider species composition and environmental factors as revealed by RDA.

442 Circles represent 289 tree-defined spider assemblages, and red crosses represent 88 species. Arrows denote environmental factors: $\mathrm{DBH}=$ Diameter at the Breast height; $\mathrm{CV}=$ Canopy Volume; $\mathrm{FC}=$ Foliage Cover.

444 Figure 4 Relative influence of environmental and spatial distance on beta diversity patterns of the spider group with 445 high (a) versus low (b) vagility. 


\section{Table $\mathbf{1}$ (on next page)}

Observed species richness, estimated species richness using non-parametric estimators (Jack1) and inventory completeness of beech (F. sylvatica) stands. 
1 Table 1 Observed species richness, estimated species richness using non-parametric estimators (Jack1) and inventory 2 completeness of beech (F. sylvatica) stands.

\begin{tabular}{lllll}
\hline & Old-grow stand & Mature stand & Young stand & All stands pooled \\
\hline Observed Richness & 55 & 66 & 51 & 88 \\
Estimated Richness & 75 & 90 & 61 & 99 \\
Inventory completeness $(\%)$ & 73.3 & 73.3 & 83.6 & 88.9 \\
\hline
\end{tabular}

3 
Figure 1

Venn diagram showing relative influence of tree architecture, spatial distance and dispersal capacity on beta diversity

(a) a conceptual representation of the contrast among environment, space, dispersal capacity and spatially-structured environmental components; (b) empirical variance partitioning of tree architecture, spatial distance, dispersal capacity effects on spider beta diversity.
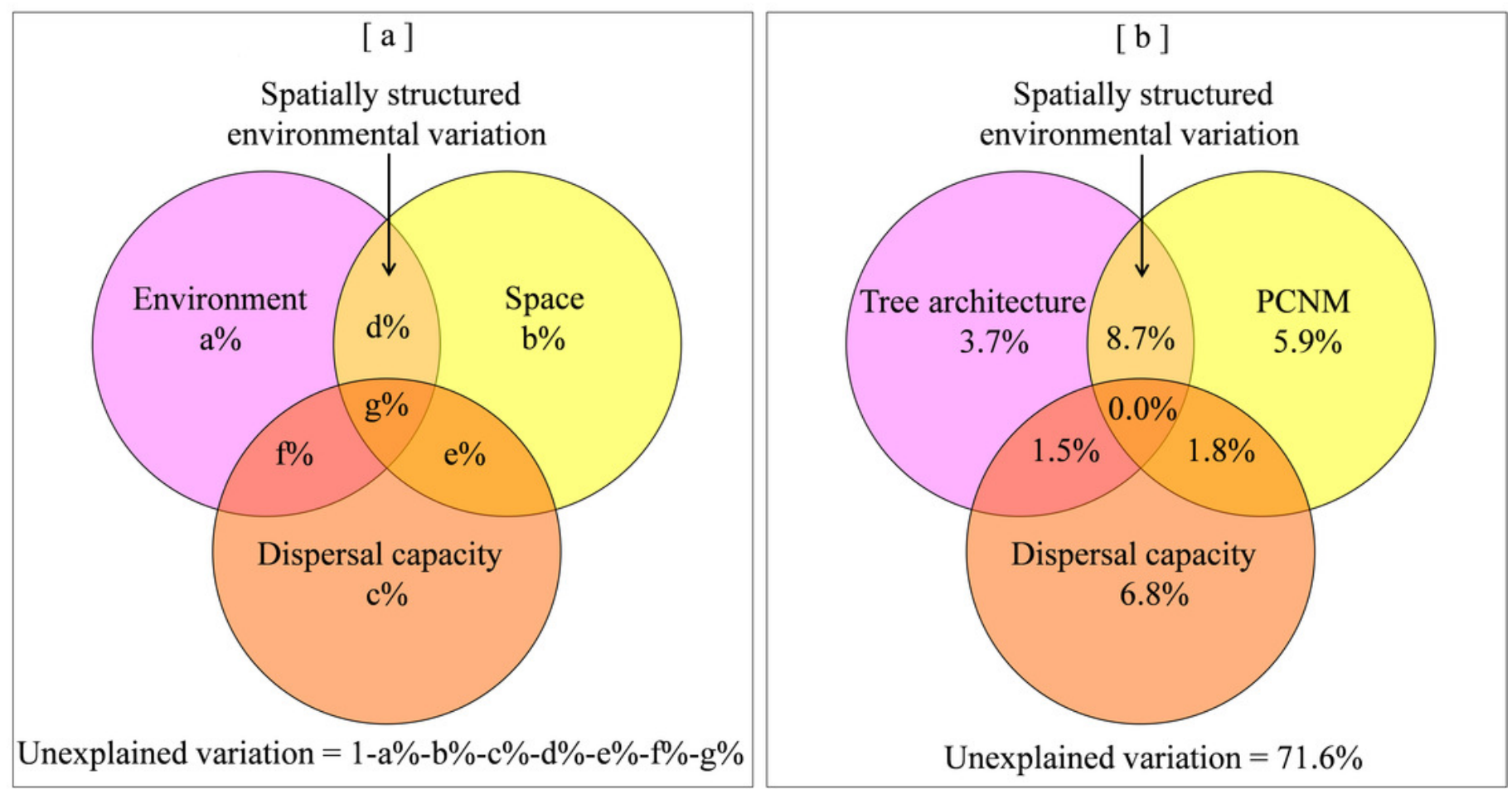
Figure 2

The standard effect size of non-random processes on spider compositional dissimilarity (i.e., Bray-Curtis index) between trees.

SES values above zero indicate that observed compositional dissimilarity is higher than expected by random assembly. The proportion of tree pairs with SES values above zero are $66.4 \%$. 


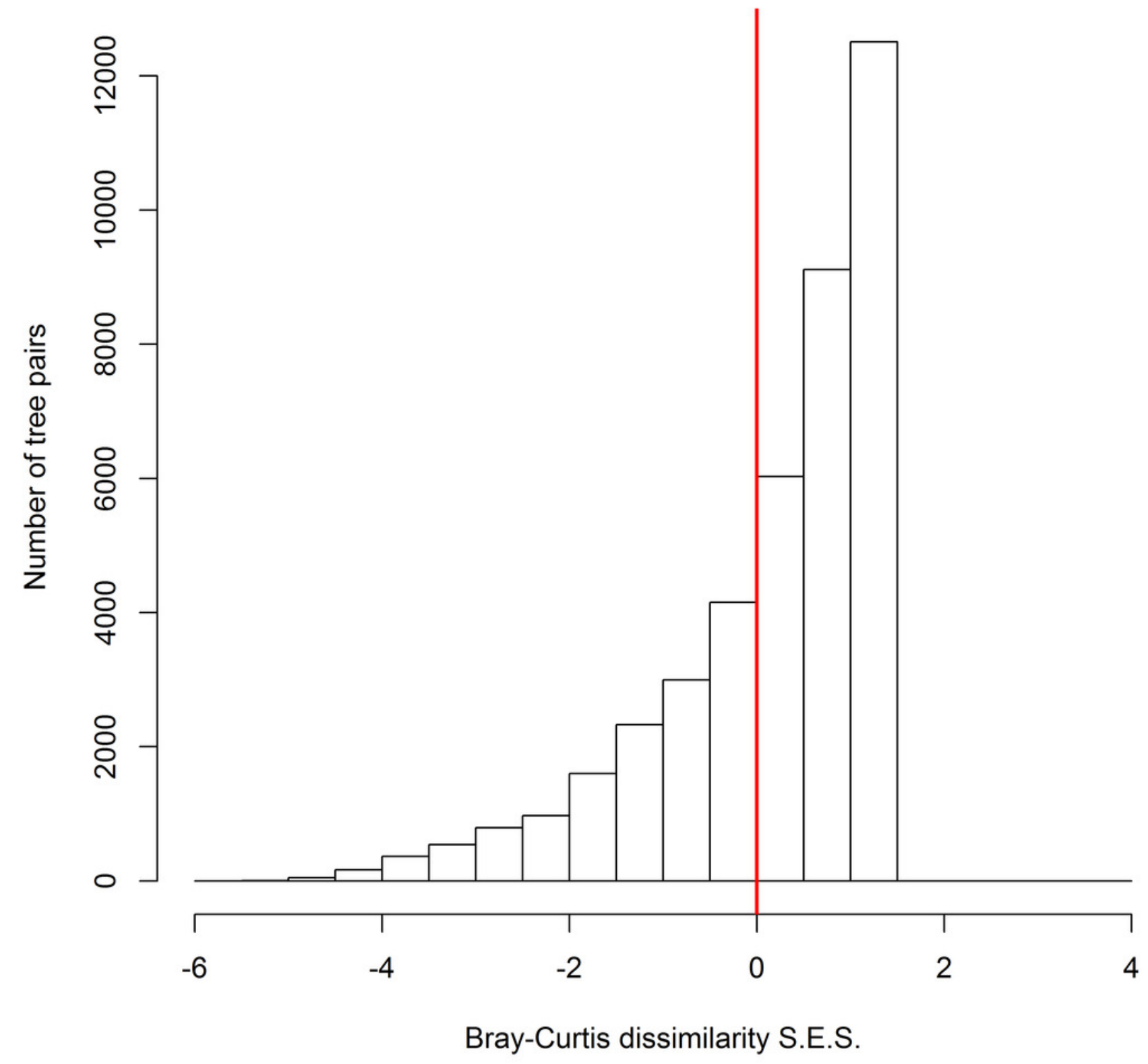




\section{Figure 3}

The relationship between spider species composition and environmental factors as revealed by RDA.

Circles represent 289 tree-defined spider assemblages, and red crosses represent 88 species. Arrows denote environmental factors: DBH=Diameter at the Breast height; $\mathrm{CV}=$ Canopy Volume; $\mathrm{FC}=$ Foliage Cover 


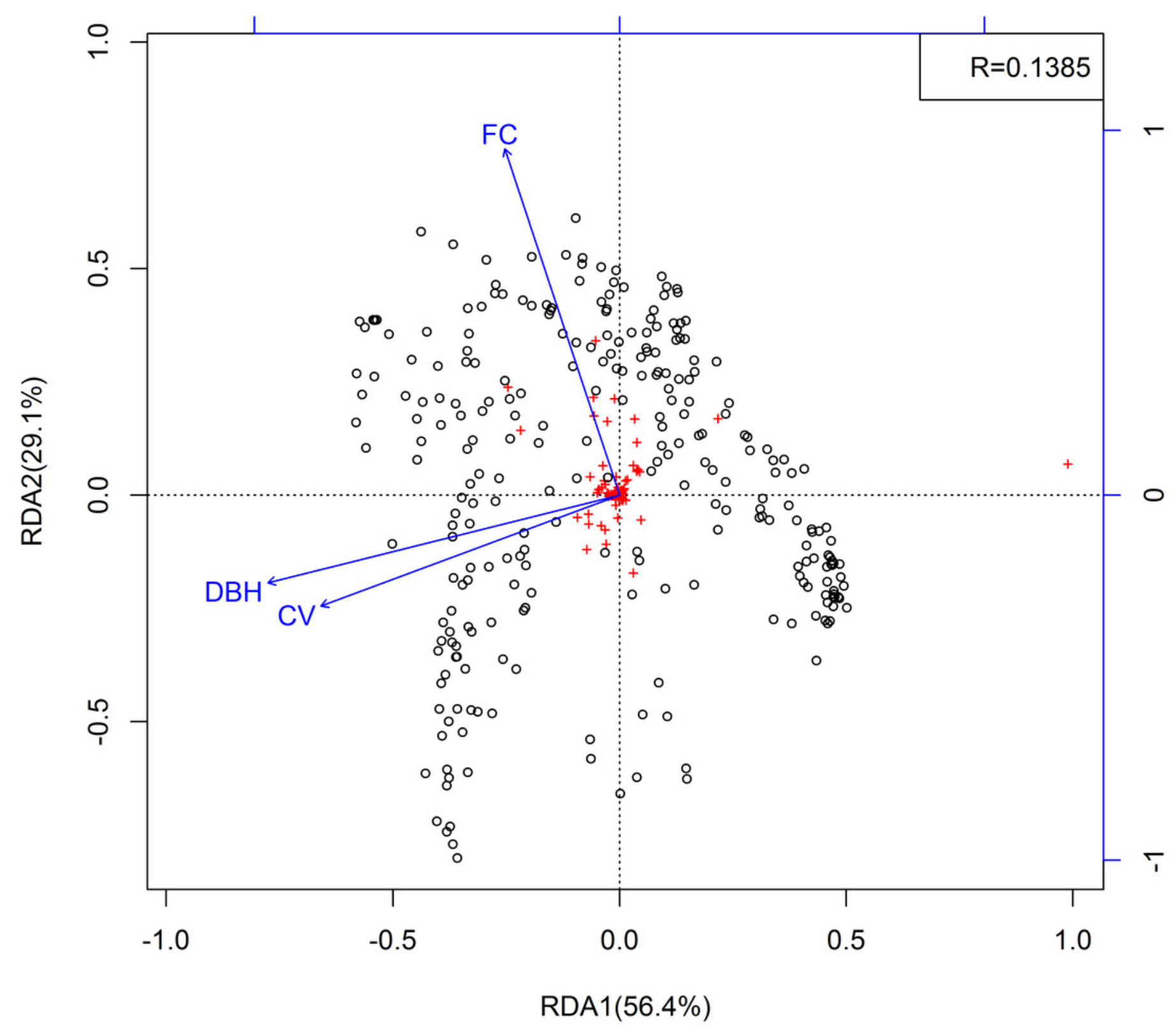




\section{Figure 4}

Relative influence of environmental and spatial distance on beta diversity patterns of the spider group with high (a) versus low (b) vagility.
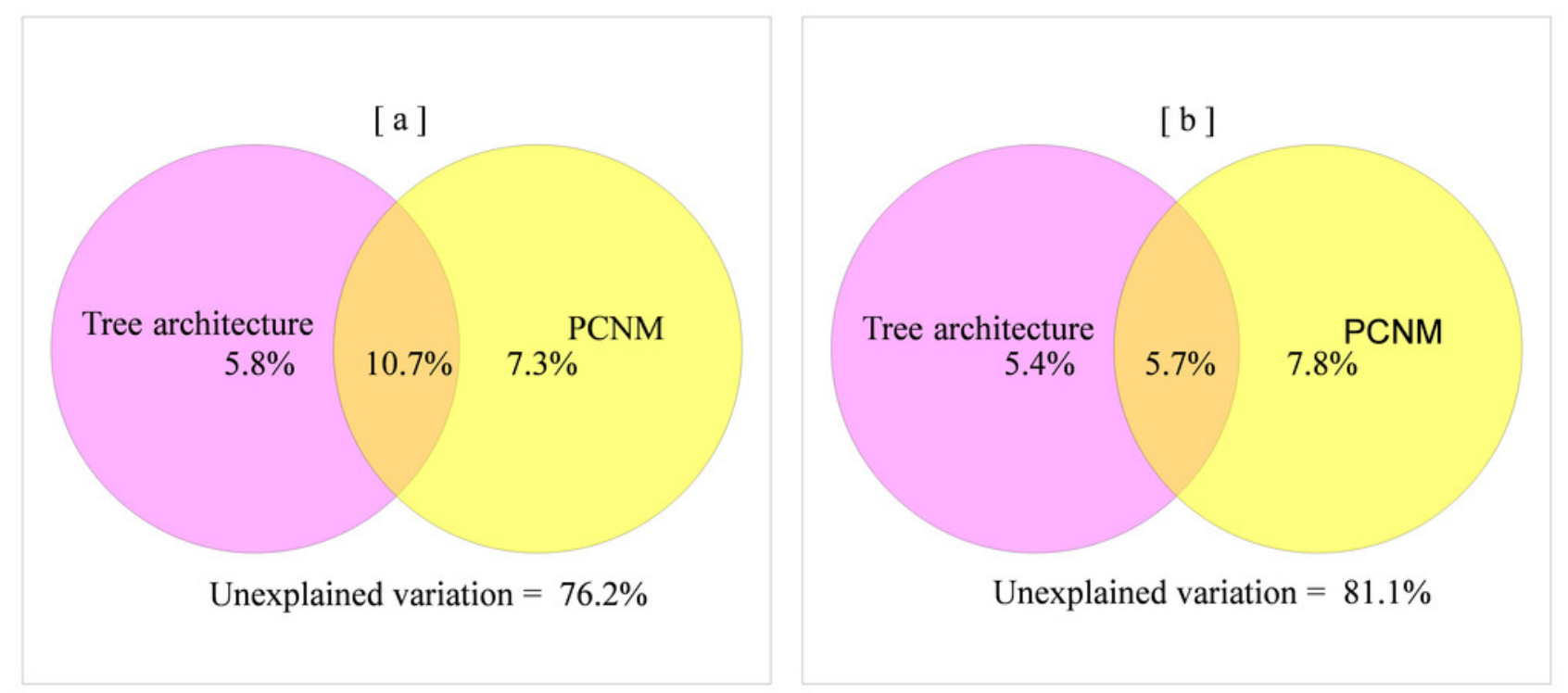